\title{
دور غرس الثقة بالنفس لدى متعلمي اللغة العربية في نجاح تعليمهم
}

Saproni

Universitas Islam Riau, Pekanbaru-Indonesia

Safroni.ahmad@edu.uir.ac.id

\begin{abstract}
ملخص
من أكبر المشاكل في تعليم اللغة العبية للمتعلمين هو الافتراض بأن اللغة العربية هي لغة صعبة التعلم، أو بعبارة أخرى، أكثر صعوبة من تعلم اللغة الأجنبية الأخرى، بحيث يواجه الطلاب قدراكبيرا من العوائق النفسية، مثل عدم الثقة بالنفس لتس من إتقان اللغة في وقت سريع نسبيا. تمدف هذه الدراسة إلى التعرف على دور غرس الثقة بالنفس لدى الطلاب في نجاح تعليم اللغة العربية. يمكننا أن نستنتج بأن الثقة بالنفس هي أمر لا يمكن الاستهانة به في تعليم اللغة العربية، لذلك يجب على المعلمين أن يأخذوا في الاعتبار أهمية غرس الثقة في طلاكمم، ويبذلوا الجهود للحفاظ على ثقة الطلاب. أما بالنسبة للمتعلمين، فيجب أن يحاولوا دائماً الخروج من منطقة عدم الثقة بالنفس عن طريق القيام بأشياء تخلق ثقة متزايدة في تعلم اللغة العربية.

\section{الكلمات المفتاحية: الثقة، نجاح التعلم، اللغة العربية}

\begin{abstract}
One of the biggest problems in learning Arabic for learners is the assumption that Arabic is a difficult language to learn, or, in other words, more difficult than learning the other foreign language, so that students face a great deal of psychological handicaps, such as lack of selfconfidence to be able to master the language In a relatively fast time. The purpose of this study is to learn about the role of instilling self-confidence for students in the success of teaching Arabic. We can conclude that self-confident is something that can not be underestimated in learning Arabic, so teachers must take into consideration the importance of instilling confidence in their students, and make efforts so that they always keep the students' confident. As for learners, they should always try to get out of the no self confidence zone by doing things that create growing confidence in learning Arabic.
\end{abstract}

Keywords: confidence, learning success, Arabic language

الخلفية

إن من أكبر المشاكل في تعلم اللغة العربية لمتعلميها هي افتراض أن اللغة العربية هي لغة صعبة للتعلم، أو بعبارة

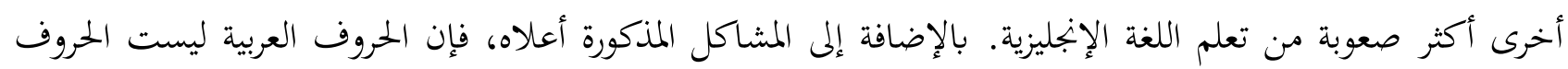

المستخدمة في كتابة أكثر اللغات المستخدمة رسميا في دول بآسيا الباسيفيكية، مما يجعل الأبجدية العربية هي الحروف أكثر

غربة مقارنة مع الحروف اللاتينية المستخدمة في كتابة اللغات المستخدة رسميا بتلك الدول. بالإضافة إلى المسألتين 
المذكورتين أعلاه، يتم تدريس اللغة العربية فيها بالصورة الأغلبية لغرض فهم الدين، وهو فهم تعاليم الدين المكتوب باللغة العربية، بجيث يرتبط تعليم اللغة العربية ارتباطا وثيقا بروح دراسة الدين. إن المشاكل المذكورة أعلاه، جعل الطلاب يواجهون إلى حد كبير بعض المعوقات النفسية، مثل عدم الثقة

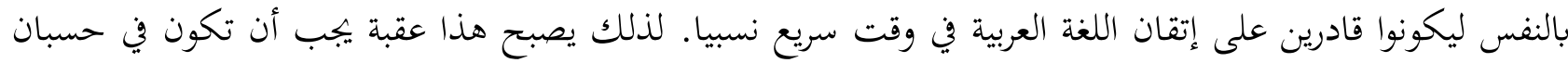
أو وعي معلمي اللغة العربية عند تقديم المواد التعليمية.

وفقا لطنطوي في قاموس مصطلحات في بجال التوجيهات والإرشادات Kamus Istilah Bimbingan Dan

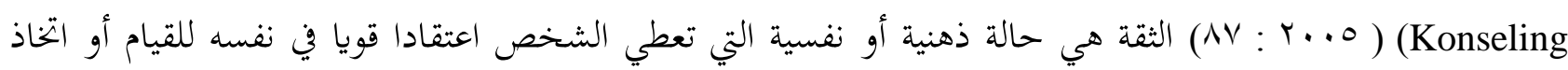
إجراءات.

ووفقا للوستر (ع . ب ؟: ع) الثقة بالنفس هي موقف أو اعتقاد شخص في قدرة الذات حتى لا توجد في أفعاله

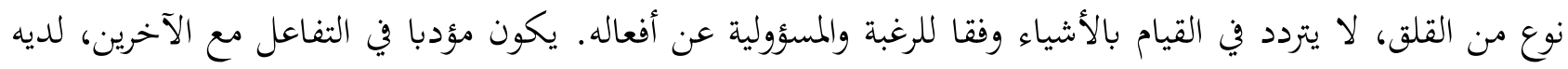

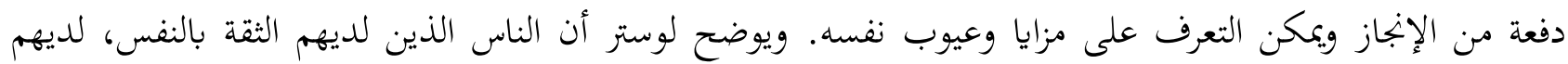

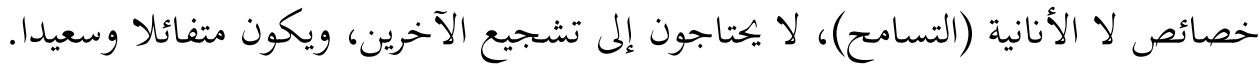
مشكلة البحث استنادا إلى وصف الخنفية أعلاه، من أجل أن تكون أكثر تركيزا وعدم توسيع المناقشة، ثم يتم تحديد صياغة المشكلة

التالية؛

1 - ما ما هو تعريف الثقة بالنفس r. با مو دور الثقة بالنفس في نجاح تعلم اللغة العربية ب. ماكيفية غرس الثقة بنفس متعلمي اللغة العربية

أهداف البحث

تمشيا مع صياغة المشكلة المذكورة أعلاه، والغرض من هذه الدراسة هو معرفة دور غرس الثقة بالنفس على الطلبة في

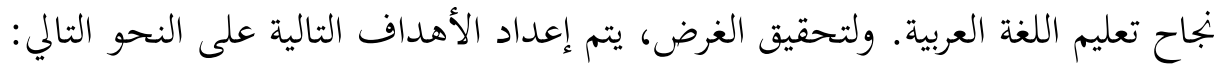
ا ـ لتحليل كيفية غرس الثقة بنغس متعلمي اللغة العربية r. التحليل دور غرس الثقة بنفس متعلمي اللغة العربية في نجاح تعليمها. فوائد البحث

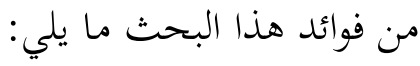
ا ـ ـ مساهمة الأفكار في تطوير استراتيجيات تعليم اللغة العربية. r. مساهمة الأفكار للمؤسسات التعليمية للعربية لتصميم منهج تدريس اللغة العربية الذي يشير إلى غرس الثقة في المتعلمين، وخاصة في المستويات الأولية. 
r. تحفيز ومساهمة الأفكار إلى مزيد من الباحثين الذين سيجرين البحوث عن المفردات العربية الواردة في لغات المتعلمين، مما يخلق انطباعا بأن اللغة العربية سهلة.

تعريفات الثقة

التعليم وفقا للقانون الإندونيسيي رقم ·r من عام r . . ب هو جهد مدروس وخخطط لتحقيق جو التعلم وعملية التعلم حتى يتمكن الطلاب من تطوير قدراهم بشكل فعال ليكون لديهم قوة روحية دينية، ضبط النفس، شخصية، ذكاء، شخصية نبيلة، ومهارات يجتاجها المجتمع والأمة والدولة. يعد تنفيذ التعليم الذي يتم تنفيذه في إندونيسيا من وقت لآخر أكثر تقليدية أو كتلة، وهو موجه نخو الكمية ليتمكن من خدمة أكبر عدد ممكن من الطلاب. الضعف الذي يظهر من تنفيذ التعليم مثل هذا هو أنه لا يلبي الاحتياجات الفردية للطلاب خارج المجموعة العادية من الطلاب. في حين أن جوهر التعليم هو تمكين الطلاب من تطوير ذكائهم ومواهبةم المحتملة على النحو الأمثل. (سوهارديتا: (1) (ب). وفقا لسوارين في (سوهارديتا: (1) (Y) تنص على أن السلوك البشري في حياته يتأثر بعاملين رئيسيين، وهما العوامل الداخلية والعوامل الخارجية. العوامل الداخلية هي كل ما يأتي من داخل الطلاب مثل: الاهتمام والذكاء، الدافع، الموقف، التفكير، الذاكرة، الثقة، الاهتمام، الموهبة والشخصية. وأما العوامل الخارجية تشمل المجتمع والأسرة والمدرسة. لذلك فإن إحدى المشكلات التي يواجهها عالم التعليم هي مشكلة تنامي الثنة بالنفس لدى الطلاب. فيما يلي بعض تعريفات الثقة بالنفس.

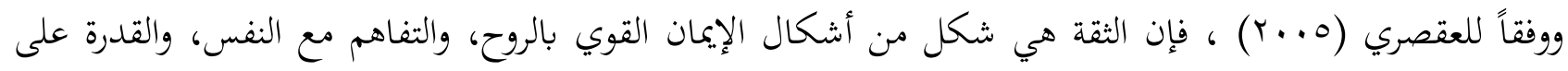
إتقان الروح. ثم أضاف العقصري أن الثقة بالنفس هي مركب بين عملية الفكر ورضا النفس. هذا يعني أننا نشعر بالرضا عن أنفسنا. أو بعبارة أخرى، فإن الموقف والسلوك الذي نظهره متجذر في مسلمة واحدة بأننا أفراداً لدينا قيم في العديد من شرائح الحياة.

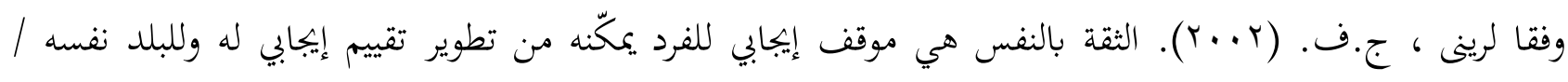
الوضع الذي يواجهه. هذا لا يعني أن الفرد قادر ومختص للقيام بكل شيء بنفسه، ويعرف أيضا باسم "sakti". إن الثقة العالية بالنفس لا تشير إلا في الواقع إلى وجود العديد من جوانب حياة الفرد حيث يشعر بأنه كفء وواثق وقادر ويؤمن بأنه قادر - لأنه مدعوم بالخبرة والإمكانيات الفعلية والإنجازات والتوقعات الواقعية لنفسه. وفقا ل (نادلر ، 11 إr) الثقة هي "معرفة قدرات الفرد وإمتلاك ما يكفي من الإيمان بها لاتخاذ قرارات سليمة في مواجهة عدم اليقين والضغط. وأضاف أن "الثقة بالنفس هي لبنة بناء للنجاح في حياته المهنية وكفاءة رئيسية في مجموعة الوعي الذاتي.

ووفقًا لسوهارديتا (1) r r) ، فإن مفهوم الثقة بالنفس هو في الأساس اعتقاد بأن يعيش حياة، وأن يفكر في الخيارات، وأن يتخذ القرارات الخاصة به وأنه قادر على القيام بشيء ما. وهذا يعني أن الثقة بالنفس لا تنشأ إلا عندما يقوم شخص ما بشيء قادر على القيام به. يشعر الشخص أساسا بالرضا عن نفسه فقط عند القيام بنشاط أو عمل أو 
توجيه قدراته. يمكن القيام بأشياء كثيرة، وهناك أيضا العديد من القدرات التي يمكن أن يتقنها شخص ما في حياته.

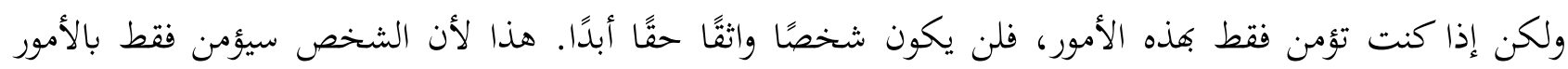
المتعلقة بما يتم فعله ويتقن بعض المهارات. الثقة هي القدرة على الثقة بالقدرات الذاتية (Perry, 2005)، الثقة بتعل الناس يتصرفون بثقة. مهما كانت التحديات التي تواجهها وتحت أي ظرف من الظروف، فإنه سيصل إلى أهدافه. الثقة هي قوة تشجع الشخص على الثعلى التقدم والتطور

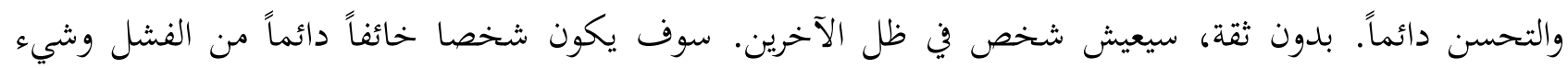
مجهول لديه.

خصائص الأفراد الذين لديهم شخصية الثقة ونقص الثقة

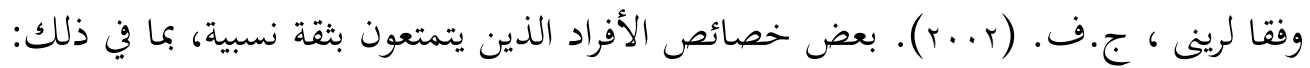

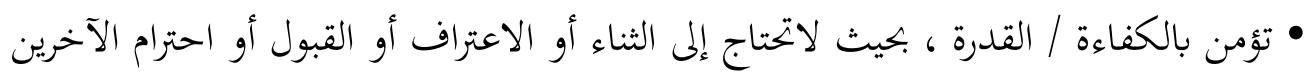

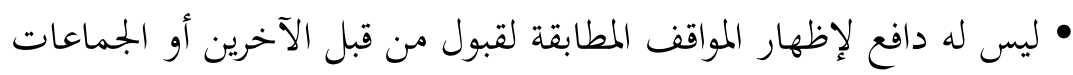

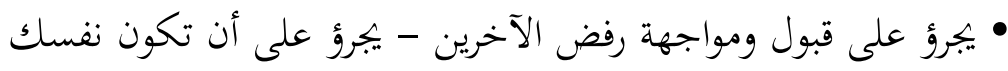

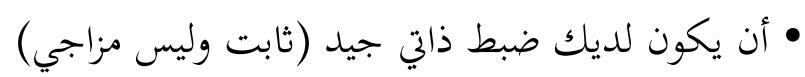
• وجود مركز داخلي للتحكم (بالنظر إلى النجاح أو الفشل، اعتمادًا على جهودك الفيد الخاصة وعدم الاستسلام بسهولة للقدر أو الظروف وعدم الاعتماد / توقع المساعدة من الآخرين)

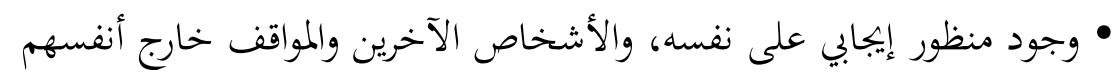

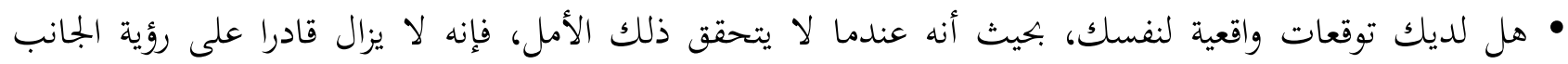
الإيجابي من نفسه والوضع.

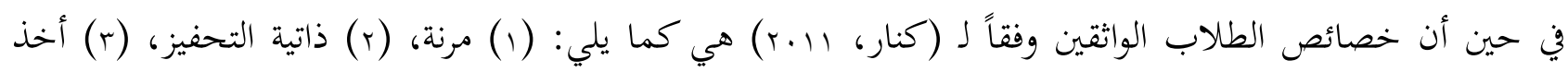

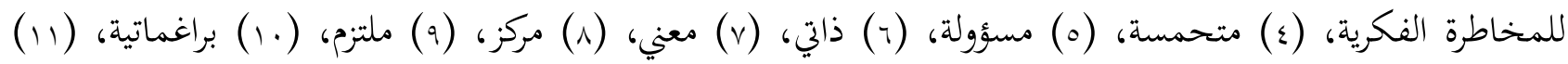

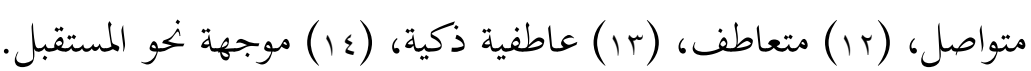

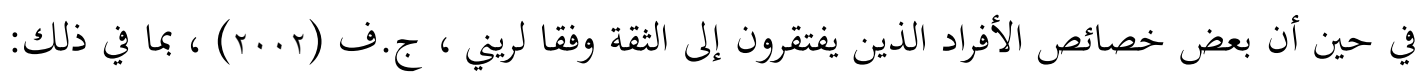

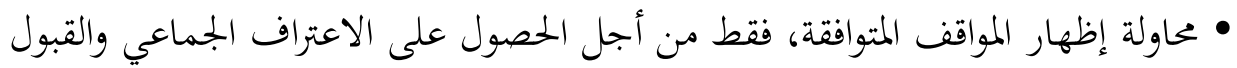

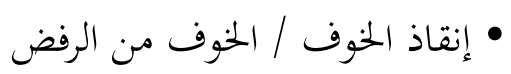
• من الصعب قبول الواقع الذاتي (خاصة قبول أوجه القصور) والنظر إلى قدراته الذاتية - ولكن من ناحية أخرى قم بتثبيت توقعات غير واقعية لنفسك

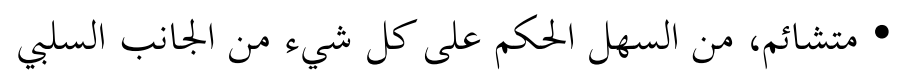

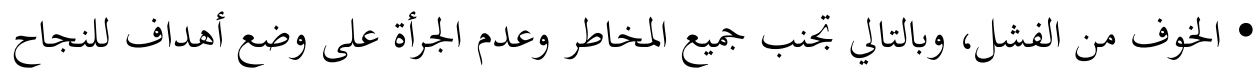


• تميل إلى رفض الثناء المقصود بإخلاص (بسبب انخفاض قيمة نفسك) • ضع / وضع نفسه دائما كآخر، لأفم يعتبرون أنفسهم غير قادرين • لديه موضع تحكم خارجي (يستسلم بسهولة للقدر، يعتمد بشكل كبير على الظروف والاعتراف / القبول ومساعدة (الآخرين)

\section{العوامل التي تؤثر على الثقة}

يقترح لوستر (ه . r) أن هناك خمسة جوانب تؤثر على ثقة الشخص بالذات، وهي (1) الاعتقاد في القدرة الذاتية، وهذا يعني موقف الشخص الإيجابي بتحاهه. (r) التفاؤل، هو موقف إيجابي من شخص لديه رؤية جيدة دائما في مواجهة كل شيء. (r) المدف، أي موقف شخص ينظر إلى كل شيء وفقا للحقيقة الصحيحة. (ء) المسؤول، أي استعداد شخص ما لتحمل كل ما أصبح نتيجة. (0) العقلانية والواقع، وهما القدرة على تحليل المشاكل أو الأحداث باستخدام أفكار معقولة. في الوقت نفسه، وفقا أنتوني (و...r)، هناك عاملان يؤثران على الثقة بالنفس. (1) العوامل الداخلية: مفهوم الذات، واحترام الذات، والحالة البدنية، والمظهر الجسدي، والفشل، والنجاح، وبتربة الحياة. (r) العوامل الخارجية: التعليم والبيئة وبتربة الحياة والعمل.

\section{علاقة الثقة بنجاح تعلّم اللغة}

فيما يتعلق بالعلاقة بين الثقة وتعلم لغة أجنبية، وفقا لكوزريني (ع ا ـ ب) استنادا إلى نتائج التحليل فمن المعروف أن هناك علاقة ثقة مع التحصيل العلمي. لا يعتمد تحسين تحصيل تعلم الطلاب على الفرد فقط. ومع ذلك، فإن التحصيل العلمي الذي يعد عاملًا خارجيًا مؤثر جدًا أيضًا. الأفراد لديهم نفس القدرة في التعلم أساسا، ولكن هناك بعض الأشياء التي تؤثر حتى يكون هناك اختلاف في تحقيق التحصيل العلمي. الطلاب الذين يواجهون مشكلة واحدة يحاول بعضهم التغلب عليها والتخلص من المشكلة، لكنهم بشكل عام لا يستطيعون التغلب عليها بمفردهم بحيث تتطلب ثقة عالية بالنفس لحل المشكلات. الثقة بالنفس هي مفتاح التحفيز الذاتي. لا يمكننا أن نعيش حياة جيدة بدون الثقة بالنفس، وهذا ما سيحتاج بشكل غير مباشر إلى الثنة بالنفس كل يوم في أشياء مختلفة، بما في ذلك تحسين تحصيل الطلاب. مستوى جيد من الثقة يسهل اتخاذ القرار ويوسع الطريق للحصول على الأصدقاء وبناء العلاقات ومساعدتنا في الحفاظ على النجاح في التعلم أو العمل بحيث يؤثر هذا بشكل غير مباشر على إنجاز الطلاب أو تحصيل الطلاب. بشكل عام، يمكن تصنيف العوامل التي تؤثر في تحصيل تعلم اللغة إلى قسمين ، هما: (1) داخلي ، وهو عامل يأتي من داخل الفرد، والذي يتضمن العوامل الفسيولوجية والعوامل النفسية، و (r) العوامل الخارجية، وهي العوامل التي تأتي من خارج الفرد، والتي تشمل العوامل الاجتماعية والعوامل غير الاجتماعية. تأتي العوامل الفسيولوجية من الحالة البدنية للشخص، وعادة ما تكون مرتبطة بشكل وثيق بالوظائف الجسدية مثل الصحة والحواس وما إلى ذلك. ترتبط العوامل

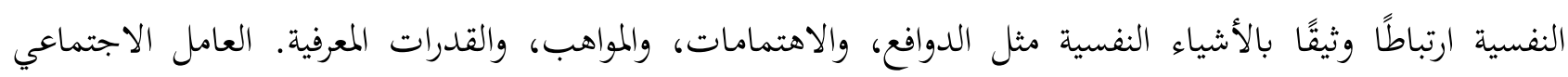
المشار إليه هنا هو العامل البشري، سواء كان الكائن البشري حاضرا أو يمكن أن يختتم وجوده. لذلك، ليس على الفور. 
يككن القول أن العوامل غير الاجتماعية لا حصر لها، على سبيل المثال: الأحوال الجوية، والهواء، وأماكن التعلم،

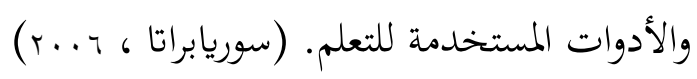

\section{دور الثقة في عملية تعلم اللغة العربية}

من نتائج الدراسات التي أجريت من قبل الباحثين، حصل على الاستنتاج بأنه كلما كان مستوى الثقة عاليا كلما كان

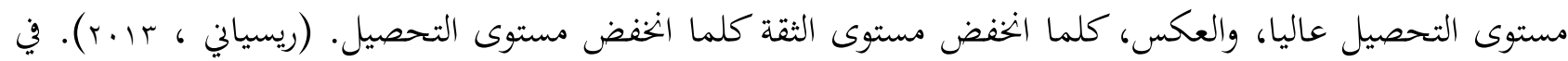

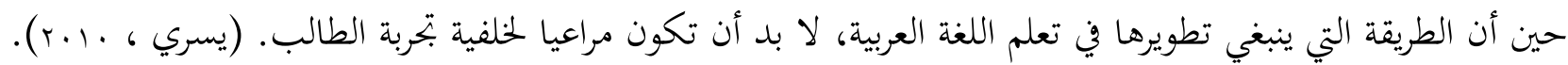

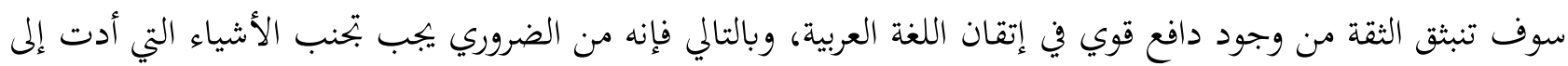

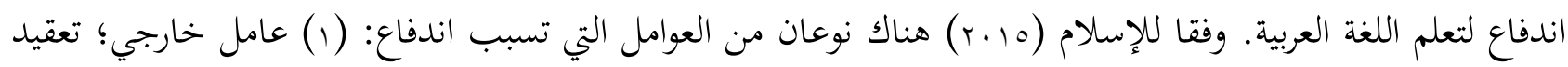

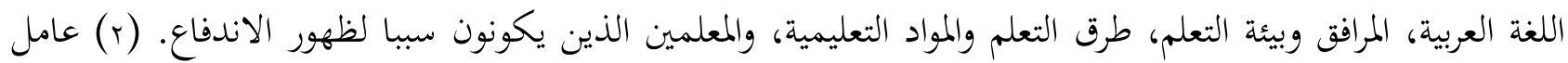
داخلي ؛ القدرات الأساسية وبحارب التعلم، المواقف السلبية تجاه اللغة العربية. كيفية تنمية الثقة للطلاب ووفقا لريني، ج.ف. (r...r) لتعزيز الثقة بالنفس، يجب أن يبدأ من داخل نفسك. من المهم جدا أن نتذكر أنه ليس هناك الشيئ يمكنه من التغلب على انعدام الثقة سوى الأفراد المعنيين. قد تكون بعض الاقتراحات التالية جديرة بالنظر

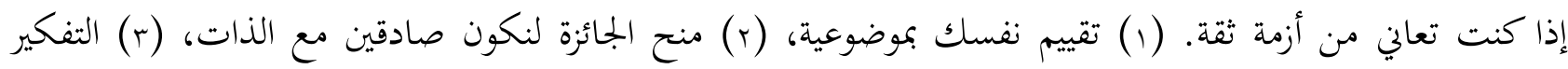

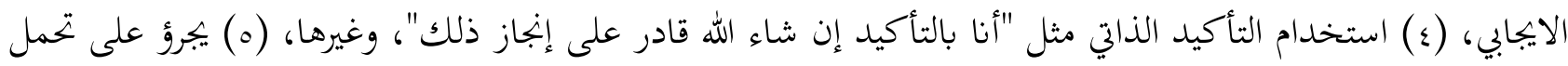

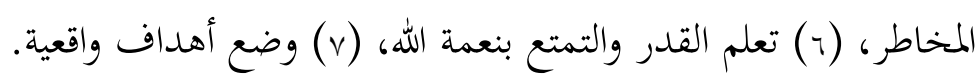

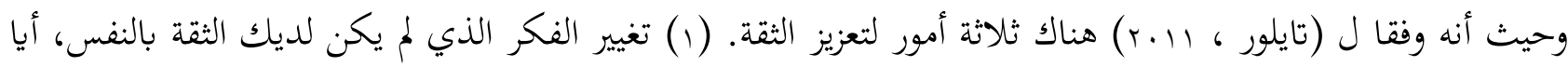

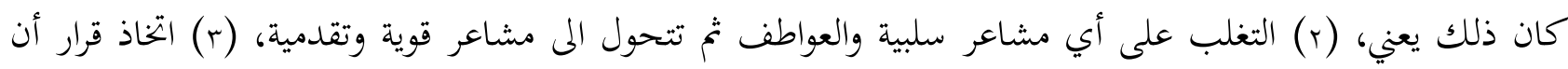
تكون مختلفا مع الآخرين. الحخلاصة والاستنتاج

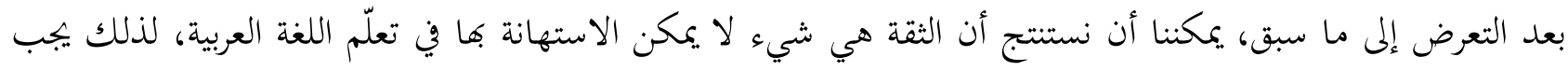

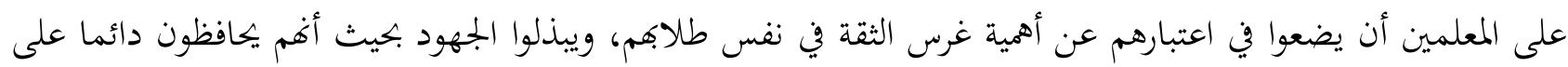

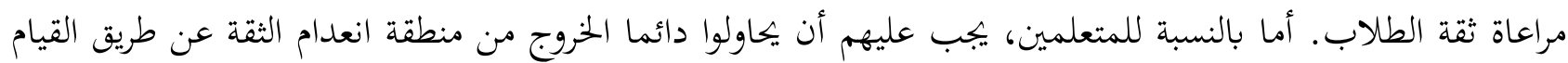
بأشياء التي تخلق الثقة المتزايدة قوية في تعلم اللغة العربية. 


\section{Bibliography}

Ahmad, S. (2018). الكتاب المدرسي لتعليم اللغة العربية للناطقين بغير ها: أهيته، ووظائه، وأهداف تعليمها. Al-Manar, 1(8). doi:http://dx.doi.org/10.24014/al-manar.v1i8.4728

Al-Uqshari, Y. (2005). Percaya Diri, Pasti! Jakarta: Gema Insasi Press.

Barbara. (2005). Percaya Diri. Dalam Barbara, Percaya Diri. Jakarta: PT. Gramedia.

Elfiky, I. (2009). Terapi berfikir Positif. Dalam I. Elfiky, Terapi berfikir Positif. Jakarta: Zaman.

Farlan, B. M., \& Fellowless, A. (2006). Are You Goog Enough (15 way to build confident minsed). London, UK: Capstone Publising Limited.

Fishe, D., \& Frey, N. (2010). Guided Instruction (How to Develop Confident and Successfull leaners). Alexandrea, USA: ASCD.

Islam, A. M. (2015). Faktor Demotivasi Pembelajaran Bahasa Arab Dalam Perspektif Siswa Madrasah. Arabiyât : Jurnal Pendidikan Bahasa Arab dan Kebahasaaraban, 1-16.

Kanar, C. C. (2011). The Confident Student. Boston, USA: Wadsword.

Kusrini, W. (2014). Hubungan Dukungan Sosial Dan Kepercayaan Diri Dengan Prestasi Bahasa Inggris siswa kelas VIII SMPN 6 Boyolali. Jurnal Penelitian Humaniora , 139.

Laskowski, L. (2006). 10 Days To More Confident Public Speaking. New York: Warner Books.

Lauster. (2002). The Personality Test. London: Pan Books.

Monart, H., \& Kase, L. (2007). The Confident Speaker. McGraw-Hill.

Nadler, R. S. (2011). Leading With Emosional Intelligence. New York: McGraw-Hill.

Naistadt, I. (2004). Speak Without Fear. Toronto: PerfectBound.

Perry, M. (2005). Confidence Boosters Pendongkrak Kepercayaan diri. Jakarta: Esensi.

Risyani, T. (2013). Pengaruh Karakter Percaya Diri terhadap Peserta didik kelas X MAN Yogyakarta 1. Yogyakarta: UIN SUKA.

Sellnow, D. D. (2005). Confident Public Speaking. Belmont, USA: Thomson Wadsword.

Suryabrata, S. (2006). Pembimbing ke Psikodiagnostik. Yogyakarta: Raka Press.

Taylor, R. (2011). Kiat-Kiat Pede. Jakarta: Gramedia.

Yusri, G. (2010). Sikap Pelajar Terhadap Pembelajaran Kemahiran Lisan Bahasa Arab Di

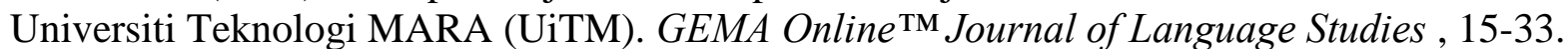

